\title{
Path Integral Quantization of Superparticle with 1/4 Supersymmetry Breaking
}

\author{
Nasser Ismail Farahat, Hanaa Abdulkareem Elegla \\ Physics Department, Islamic University of Gaza, Gaza, Palestine \\ Email: nfarahat@iugaza.edu.ps, helegla@iugaza.edu.ps
}

Received September 28, 2013; revised October 28, 2013; accepted November 5, 2013

Copyright (C) 2013 Nasser Ismail Farahat, Hanaa Abdulkareem Elegla. This is an open access article distributed under the Creative Commons Attribution License, which permits unrestricted use, distribution, and reproduction in any medium, provided the original work is properly cited.

\begin{abstract}
We present path integral quantization of a massive superparticle in $d=4$ which preserves $\frac{1}{4}$ of the target space supersymmetry with eight supercharges, and so corresponds to the partial breaking $N=8 \rightarrow N=2$. Its worldline action contains a Wess-Zumino term, explicitly breaks $d=4$ Lorentz symmetry and exhibits one complex fermionic $\kappa$-symmetry. We perform the Hamilton-Jacobi formalism of constrained systems, to obtain the equations of motion of the model as total differential equations in many variables. These equations of motion are in exact agreement with those obtained by Dirac's method.
\end{abstract}

Keywords: Hamilton-Jacobi Formalism; Singular Lagrangian; Supersymmetry

\section{Introduction}

The theory of constrained systems is a basis of modern physics: gauge field theories, quantum gravity, supergravity, string and superstring models are examples of systems with constraints. For such theories, one should specify not only an evolution equation but also additional requirements (constraints) being imposed on initial conditions [1].

A standard consistent way of dealing with singular systems was first formulated by Dirac [2]. In Dirac formulism, when a singular Lagrangian in configuration space is transformed into a singular Lagrangian in phase space, the inherent constraints would be generated, which are called Dirac primary constraints [3-5]. Through the consistency conditions, step by step, using these primary constraints may generate more new inherent constraints, which are called Dirac secondary constraints. Such a way to calculate different constraints in Dirac formalism is named as Dirac-Bergmann algorithm, which was first proposed by Bergmann [6,7].

Canonical path integral method is a kind of quantization method [8,9], which depends on Hamilton-Jacobi formalism shown by Güler [10,11]. This method has some very useful properties of obviating the need to distinguish primary and secondary constraints and the first and the second types of constraints. The method is simpler, and does not have such a hypothesis of Diracs conjecture, thus it has evoked much attention [12-20].

Partial breaking of global supersymmetry (PBGS) [2124] is widely understood to be an inborn feature of supersymmetric extended objects. The concept of PBGS provides a manifestly off-shell supersymmetric worldvolume description of various superbranes in terms of Goldstone superfields $\psi^{i}(x), \bar{\psi}^{i}(x)$. The physical worldvolume multiplets of the given superbrane are interpreted as Goldstone superfields realizing the spontaneous breaking of the full brane supersymmetry group down to its unbroken worldvolume subgroup $[25,26]$. The technical tools here are the method of nonlinear realizations [27-29]. Recently, there has been a growing interest in PBGS options other than the $1 / 2$ breaking [30-34]. This is essentially due to the discovery of the $d=11$ supergravity solutions preserving $1 / 4$ or $1 / 8$ of the $d=11$ supersymmetry [30] and their subsequent interpretation in terms of intersecting branes. Since branelike worldvolume effective actions which would be capable of describing those solutions are still unknown, it seems interesting to study pointlike models that mimic the exotic supersymmetry breaking options inherent in the intersecting branes. Such models could share some character- 
istic features of the systems of intersecting branes, much like the ordinary superparticle bears a similarity to the Green-Schwarz superstring. Superparticle models exhibiting $3 / 4$ or $1 / 4$ PBGS have been constructed [35-38]. The $1 / 4$ breaking of the original $N=8$ supersymmetry down to $N=2$ manifests itself in the presence of only one complex-symmetry in the corresponding worldline action. This is achieved at the cost of the explicit breaking of the target space Lorentz symmetry down to $\mathrm{SO}(3)$ symmetry (in the fermionic sector).

In the present paper we study the canonical path integral quantization for $N=8 \rightarrow N=2$ model as a typical example of massive superparticles with 1/4 PBGS in an ordinary four dimensional Minkowski spacetime (with $R^{4 / 8}$ as the target superspace and explicitly broken Lorentz symmetry) [39]. Prior to quantization, Hamiltonian analysis is accomplished in full detail, by obtaining the set of inherent constraints, and the equations of motion as total differential equations. Our paper is organized as follows. Hamilton-Jacobi formulation is presented in Section 2. In Section 3, the canonical path integral quantization of our model is investigated. In Section 4 , the conclusion is given.

\section{Hamilton-Jacobi Formalism of Constrained Systems}

The system that is described by the Lagrangian $L\left(q_{i}, \dot{q}_{i}, t\right), \quad i=1, \cdots, n$, is constrained system if the Hessian matrix

$$
A_{i j}=\frac{\partial^{2} L}{\partial \dot{q}_{i} \partial \dot{q}_{j}} \quad i, j=1, \cdots, n,
$$

has a rank $(n-r), r<n$. In this case we have $r$ momenta are dependent of each other. The generalized momenta $p_{i}$ corresponding to the generalized coordinates $q_{i}$ are defined as,

$$
\begin{aligned}
& p_{a}=\frac{\partial L}{\partial \dot{q}_{a}} \quad a=1, \cdots, n-r, \\
& p_{\mu}=\frac{\partial L}{\partial \dot{q}_{\mu}} \quad \mu=n-r+1, \cdots, n .
\end{aligned}
$$

Since the rank of the Hessian matrix is $(n-r)$, one may solve (2) for $\dot{q}_{a}$ as

$$
\dot{q}_{a}=\omega_{a}\left(q_{i}, \dot{q}_{\mu}, p_{a}\right) .
$$

Substituting (4) into (3), we obtain relations in $q_{i}, p_{a}, \dot{q}_{v}$ and $t$ in the form

$$
p_{\mu}=\left.\frac{\partial L}{\partial \dot{q}_{\mu}}\right|_{\dot{q}_{a}=\omega_{a}}=-H_{\mu}\left(q_{i}, \dot{q}_{\nu}, \dot{q}_{a}=\omega_{a}, p_{a}, t\right) .
$$

The canonical Hamiltonian $H_{0}$ is defined as

$$
H_{0}=-L\left(q_{i}, \dot{q}_{\nu}, \dot{q}_{a}=\omega_{a}, t\right)+p_{a} \omega_{a}+\left.\dot{q}_{\mu} p_{\mu}\right|_{p_{v}=-H_{v}} .
$$

The set of Hamilton-Jacobi partial differential equations (HJPDE) is expressed as

$$
\begin{array}{r}
H_{\alpha}^{\prime}\left(q_{\beta} ; q_{a} ; p_{a}=\frac{\partial S}{\partial q_{a}} ; p_{\alpha}=\frac{\partial S}{\partial q_{\alpha}}\right)=0, \\
\alpha, \beta=0, n-r+1, \cdots, n,
\end{array}
$$

where

$$
\begin{aligned}
& H_{0}^{\prime}=p_{0}+H_{0} ; \\
& H_{\mu}^{\prime}=p_{\mu}+H_{\mu} .
\end{aligned}
$$

with $q_{0}=t$ and $S$ being the action. The equations of motion are obtained as total differential equations in many variables such as,

$$
\begin{gathered}
\mathrm{d} q_{a}=\frac{\partial H_{\alpha}^{\prime}}{\partial p_{a}} \mathrm{~d} t_{\alpha}, \\
\mathrm{d} p_{\beta}=\frac{\partial H_{\alpha}^{\prime}}{\partial q_{\beta}} \mathrm{d} t_{\alpha}, \\
\mathrm{d} Z=\left(-H_{\alpha}+p_{a} \frac{\partial H_{\alpha}^{\prime}}{\partial p_{a}}\right) \mathrm{d} t_{\alpha} .
\end{gathered}
$$

where $Z=S\left(t_{\alpha}, q_{a}\right)$.These equation are integrable if and only if

$$
\begin{gathered}
\mathrm{d} H_{0}^{\prime}=0, \\
\mathrm{~d} H_{\mu}=0, \quad \mu=n-r+1, \cdots, n .
\end{gathered}
$$

If the conditions (13) and (14) are not satisfied identically, we consider them as new constraints and we examine their variations. Thus repeating this procedure, one may obtain a set of constraints such that all the variations vanish, then we may solve the equations of motion (10) and (11) to get the canonical phase-space coordinates as

$$
q_{a} \equiv q_{a}\left(t, t_{\mu}\right), \quad p_{a} \equiv p_{a}\left(t, t_{\mu}\right), \quad \mu=1, \cdots, r .
$$

In this case the path integral representation may be written as

$$
\begin{gathered}
\langle\text { Out }|S| \mathrm{In}\rangle \\
=\int \prod_{a=1}^{n-r} \mathrm{~d} q^{a} \mathrm{~d} p^{a} \exp \left[i \int_{t_{\alpha}}^{t_{\alpha}^{\prime}}\left(-H_{\alpha}+p_{a} \frac{\partial H_{\alpha}^{\prime}}{\partial p_{a}}\right) \mathrm{d} t_{\alpha}\right], \\
a=1, \cdots, n-r, \quad \alpha=0, n-r+1, \cdots, n .
\end{gathered}
$$

We should notice that the integral (16) is an integration over the canonical phase space coordinates $\left(q_{a}, p_{a}\right)$.

\section{Hamilton-Jacobi Formulation of Superparticle with 1/4 Supersymmetry Breaking}

The action functional of a massive superparticle model exhibiting 1/4 PBGS, is written as [39] 


$$
S=\int \mathrm{d} \tau\left\{\frac{1}{2 e}\left(-\Pi^{0} \Pi^{0}+\Pi^{i} \Pi^{i}\right)-\frac{1}{2} e m^{2}+i m\left(\theta \dot{\bar{\theta}}-\psi^{i} \dot{\bar{\psi}}^{i}\right)\right\} .
$$

where

$$
\begin{aligned}
& \Pi^{0}=\dot{x}^{0}+\frac{i}{2} \theta \dot{\bar{\theta}}+\frac{i}{2} \bar{\theta} \dot{\theta}+\frac{i}{2} \psi^{i} \dot{\bar{\psi}}^{i}+\frac{i}{2} \bar{\psi}^{i} \dot{\psi}^{i}, \\
& \Pi^{i}=\dot{x}^{i}+i \psi^{i} \dot{\theta}+i \bar{\psi}^{i} \dot{\bar{\theta}}, \quad i=1,2,3 .
\end{aligned}
$$

and $\theta, \psi^{i}$ are four complex fermions parameterizing the odd sector of the model. The Lagrangian is

$$
L=\frac{1}{2 e}\left(-\Pi^{0} \Pi^{0}+\Pi^{i} \Pi^{i}\right)-\frac{1}{2} e m^{2}+i m\left(\theta \dot{\bar{\theta}}-\psi^{i} \dot{\bar{\psi}}^{i}\right),
$$

The singularity of the the Lagrangian follows from the fact that the rank of the Hessian matrix $A_{i j}$ is two.

The canonical momenta defined in (2) and (3) read as

$$
\begin{aligned}
& P^{0}=\frac{\partial L}{\partial \dot{x}^{0}}=-\frac{1}{e}\left(\dot{x}^{0}+\frac{i}{2} \theta \dot{\bar{\theta}}+\frac{i}{2} \bar{\theta} \dot{\theta}+\frac{i}{2} \psi^{i} \dot{\bar{\psi}}^{i}+\frac{i}{2} \bar{\psi}^{i} \dot{\psi}^{i}\right), \\
& P^{i}=\frac{\partial L}{\partial \dot{x}^{i}}=\frac{1}{e}\left(\dot{x}^{i}+i \psi^{i} \dot{\theta}+i \bar{\psi}^{i} \dot{\bar{\theta}}\right), \\
& \pi_{\theta}=\frac{\partial L}{\partial \dot{\theta}}=\frac{i}{2}\left(P^{0}+m\right) \bar{\theta}+i P^{i} \psi^{i}=-H_{\theta}, \\
& \bar{\pi}_{\bar{\theta}}=\frac{\partial L}{\partial \dot{\dot{\theta}}}=\frac{i}{2}\left(P^{0}+m\right) \theta+i P^{i} \bar{\psi}^{i}=-H_{\bar{\theta}}, \\
& \pi_{\psi}^{i}=\frac{\partial L}{\partial \dot{\psi}^{i}}=\frac{i}{2}\left(P^{0}-m\right) \bar{\psi}^{i}=-H_{\psi^{i}}, \\
& \bar{\pi}_{\bar{\psi}}^{i}=\frac{\partial L}{\partial \dot{\bar{\psi}}^{i}}=\frac{i}{2}\left(P^{0}-m\right) \psi^{i}=-H_{\bar{\psi}^{i}},
\end{aligned}
$$

and

$$
P_{e}=\frac{\partial L}{\partial \dot{e}}=0=-H_{e} .
$$

Now the velocities $\dot{X}^{0}$ and $\dot{X}^{i}$ can be expressed in terms of the momenta $P^{0}$ and $P^{i}$ respectively as

$$
\dot{x}^{0}=-\left(e P^{o}+\frac{i}{2} \theta \dot{\bar{\theta}}+\frac{i}{2} \bar{\theta} \dot{\theta}+\frac{i}{2} \psi^{i} \dot{\bar{\psi}}^{i}+\frac{i}{2} \bar{\psi}^{i} \dot{\psi}^{i}\right) .
$$

and

$$
\dot{x}^{i}=\left(e P^{i}-i \psi^{i} \dot{\theta}-i \bar{\psi}^{i} \dot{\bar{\theta}}\right) .
$$

The canonical Hamiltonian $H$ is obtained as

$$
H=\frac{1}{2} e\left(-P^{0} P^{0}+P^{i} P^{i}+m^{2}\right) .
$$

The set of HJPDE's are

$$
H^{\prime}=P+\frac{1}{2} e\left(-P^{0} P^{0}+P^{i} P^{i}+m^{2}\right)=0,
$$

$$
\begin{aligned}
& H_{\theta}^{\prime}=\pi_{\theta}-\frac{i}{2}\left(P^{0}+m\right) \bar{\theta}-i P^{i} \psi^{i}=0, \\
& H_{\bar{\theta}}^{\prime}=\bar{\pi}_{\bar{\theta}}-\frac{i}{2}\left(P^{0}+m\right) \theta-i P^{i} \bar{\psi}^{i}=0, \\
& H_{\psi^{i}}^{\prime}=\pi_{\psi}^{i}-\frac{i}{2}\left(P^{0}-m\right) \bar{\psi}^{i}=0, \\
& H_{\bar{\psi}^{i}}^{\prime}=\bar{\pi}_{\bar{\psi}}^{i}-\frac{i}{2}\left(P^{0}-m\right) \psi^{i}=0,
\end{aligned}
$$

and

$$
H_{e}^{\prime}=P_{e}=0 .
$$

Therefore, the total differential equations for the characteristics read as

$$
\mathrm{d} x^{0}=-e P^{o} \mathrm{~d} \tau-\frac{i}{2} \theta \mathrm{d} \bar{\theta}-\frac{i}{2} \bar{\theta} \mathrm{d} \theta-\frac{i}{2} \psi^{i} \mathrm{~d} \bar{\psi}^{i}-\frac{i}{2} \bar{\psi}^{i} \mathrm{~d} \psi^{i},
$$

$$
\begin{aligned}
& \mathrm{d} x^{i}=e P^{i} \mathrm{~d} \tau-i \psi^{i} \mathrm{~d} \theta-i \bar{\psi}^{i} \mathrm{~d} \bar{\theta}, \\
& \mathrm{d} P^{0}=0, \\
& \mathrm{~d} P^{i}=0, \\
& \mathrm{~d} \pi_{\theta}=\frac{i}{2}\left(P^{0}+m\right) \mathrm{d} \bar{\theta}, \\
& \mathrm{d} \bar{\pi}_{\bar{\theta}}=\frac{i}{2}\left(P^{0}+m\right) \mathrm{d} \theta, \\
& \mathrm{d} \pi_{\psi}^{i}=i P^{i} \mathrm{~d} \theta+\frac{i}{2}\left(P^{0}-m\right) \mathrm{d} \bar{\psi}^{i}, \\
& \mathrm{~d} \bar{\pi}_{\bar{\psi}}^{i}=i P^{i} \mathrm{~d} \bar{\theta}+\frac{i}{2}\left(P^{0}-m\right) \mathrm{d} \psi^{i},
\end{aligned}
$$

and

$$
\mathrm{d} P_{e}=-\frac{1}{2}\left(-P^{0} P^{0}+P^{i} P^{i}+m^{2}\right) \mathrm{d} \tau .
$$

To check whether the set of Equations (35)-(43) are integrable or not, let us consider the total variations of the set of (HJPDE)'s. The variation of

$$
\begin{aligned}
\mathrm{d} H^{\prime} & =0, \\
\mathrm{~d} H_{\theta}^{\prime} & =0, \\
\mathrm{~d} H_{\bar{\theta}}^{\prime} & =0, \\
\mathrm{~d} H_{\psi^{i}}^{\prime} & =0, \\
\mathrm{~d} H_{\bar{\psi}^{i}}^{\prime} & =0,
\end{aligned}
$$

are identically zero, whereas

$$
\mathrm{d} H_{e}^{\prime}=\frac{1}{2}\left(-P^{0} P^{0}+P^{i} P^{i}+m^{2}\right) \mathrm{d} \tau=H_{e}^{\prime \prime} \mathrm{d} t .
$$

where 


$$
H_{e}^{\prime \prime}=\frac{1}{2}\left(-P^{0} P^{0}+P^{i} P^{i}+m^{2}\right)=0 .
$$

is a new constraint. Thus the equations of motion (35)(43) and the new constraint (50) represent an integrable system.

Now to obtain the path integral quantization of this system, we can use (12) to obtain the canonical action integral as

$$
S=\int\left\{\frac{1}{2} e\left(-P^{0} P^{0}+P^{i} P^{i}\right)-\frac{1}{2} e m^{2}+i m\left(\theta \dot{\bar{\theta}}-\psi^{i} \dot{\bar{\psi}}^{i}\right)\right\} \mathrm{d} \tau .
$$

By using (51) and (16) the path integral for the system is expressed as

$$
\begin{aligned}
& \left\langle x^{0}, x^{i}, \tau ; x^{\prime 0}, x^{i}, \tau^{\prime}\right\rangle \\
& =\int \mathrm{d} x^{0} \mathrm{~d} x^{i} \mathrm{~d} P^{0} \mathrm{~d} P^{i} \exp \left[i \int \left\{\frac{1}{2} e\left(-P^{0} P^{0}+P^{i} P^{i}\right)\right.\right. \\
& \left.\left.\quad-\frac{1}{2} e m^{2}+i m\left(\theta \dot{\bar{\theta}}-\psi^{i} \dot{\bar{\psi}}^{i}\right)\right\} \mathrm{d} \tau\right]
\end{aligned}
$$

\section{Conclusion}

The path integral qantization of constrained systems is obtained for using the canonical path integral method, which based on the constrained Hamilton theory. The equations of motion are obtained as total differential equations in many variables, and the integrability conditions were shown to be equivalent to the vanishing of the variation of each $H_{\beta}^{\prime}$, i.e. $\mathrm{d} H_{\alpha}^{\prime}=0$ 's, then the system is integrable. In this paper, we examined Hamiltonian treatment of a massive superparticle model with $1 / 4$ partial breaking of global supersymmetry which propagates in four dimensional flat spacetime. We obtain constraints in phase space, which contains all kinds of constraints (primary and secondary, first and second class ones). This example is very illustrative, since it allows a comparison between all features of Diracs and Hamilton-Jacobi formalisms. In Dirac's formalism, we must reduce any constrained singular system to one with firstclass constraints only, and we must call attention to the presence of arbitrary variables in some of the Hamiltonian equations of motion due to the fact that we have gauge dependent variables, therefore we have made a gauge fixing. This does not occur in Hamilton-Jacobi formalism since it provides a gauge-independent description of the systems evolution due to the fact that the Hamilton-Jacobi function S contains all the solutions that are related by gauge transformations. Our results are in agreement with those given in Dirac's method [39].

\section{REFERENCES}

[1] M. Henneaux and C. Teitelboim, "Quantization of Gauge
Systems,” Princeton University Press, New Jersey, 1992.

[2] P. A. M. Dirac, "Lectures on Quantum Mechanics (Belfer Graduate School of Science)," Yeshiva University, New York, 1964.

[3] K. Sundermeyer, "Lecture Notes in Physics," Spring-Verlag, Berlin, 1982.

[4] D. M. Gitman and I. V. Tyutin, "Quantization of Fields with Constraints,” Springer-Verlag, Berlin, 1990. http://dx.doi.org/10.1007/978-3-642-83938-2

[5] J. Govaerts, "Hamiltonian Quantisation and Constrsined Dynamics,” Vol. 4, Leuven University Press, 1991.

[6] J. L. Anderson and P. G. Bergmann, "Constraints in Covariant Field Theories," Physical Review, Vol. 83, No. 5, 1951, pp. 1018-1025. http://dx.doi.org/10.1103/PhysRev.83.1018

[7] P. G. Bergmann and J. Goldberg, "Dirac Bracket Transformations in Phase Space," Physical Review, Vol. 98, No. 2, 1955, pp. 531-538. http://dx.doi.org/10.1103/PhysRev.98.531

[8] S. I. Muslih, "Path Integral Quantization of Electromagnetic Theory," Nuovo Cimento B, Vol. 115, No. 1, 2000, p. 7.

[9] S. I. Muslih, "Quantization of Parametrization Invariant Theories," Nuovo Cimento B, Vol. 115, 2002, p. 1.

[10] Y. Güler, "Integration of Singular Systems," Nuovo Cimento B, Vol. 107, No. 10, 1992, pp. 1143-1149. http://dx.doi.org/10.1007/BF02727199

[11] Y. Güler, "Canonical Formulation of Constrained Systems,” Nuovo Cimento B, Vol. 107, No. 12, 1992, pp. 13891395. http://dx.doi.org/10.1007/BF02722849

[12] N. I. Farahat and Y. Güler, "Treatment of a Relativistic Particle in External Electromagnetic Field as a Singular System," Nuovo Cimento B, Vol. 111, No. 4, 1996, pp. 513-520. http://dx.doi.org/10.1007/BF02724560

[13] E. M. Rabei and S. Tawfiq, "Hamilton-Jacobi Treatment of QED and Yang-Mills Theory as Constrained Systems," Hadronic Journal, Vol. 20, 1997, p. 232.

[14] S. I. Muslih, "Canonical Path Integral Quantization of Einstein's Gravitational Field," General Relativity and Gravitation, Vol. 34, No. 7, 2002, pp. 1059-1065.

http://dx.doi.org/10.1023/A:1016561904569

[15] N. I. Farahat and Z. Nassar, "Relativistic Classical Spinning Particle as Singular System of Second Order," Islamic University Journal, Vol. 13, 2005, p. 239.

[16] N. I. Farahat and Z. Nassar, "Treatment of a Spinning Particle or Super-gravity in One Dimension Singular System,” Hadronic Journal, Vol. 25, 2002, p. 239.

[17] S. I. Muslih and Y. Güler, "Is Gauge Fixing of Constrained Systems Necessary?” Nuovo Cimento B, Vol. 113, 1998, p. 277.

[18] S. I. Muslih and Y. Güler, "The Feynman Path Integral Quantization of Constrained Systems," Nuovo Cimento B, Vol. 112, 1997, p. 97.

[19] S. I. Muslih, "Reduced Phase-Space Quantization of Constrained Systems," Nuovo Cimento B, Vol. 117, No. 4, 2002, p. 383. 
[20] N. I. Farahat and H. A. Elegla, "Hamilton-Jacobi Formulation of Siegle Superparticle,” Turkish Journal of Physics, Vol. 30, No. 6, 2006, pp. 473-478.

[21] J. Bagger and J. Wess, "Partial Breaking of Extended Supersymmetry,” Physics Letters B, Vol. 138, No. 1-3, 1984, pp. 105-110. http://dx.doi.org/10.1016/0370-2693(84)91882-3

[22] J. Hughes, J. Liu and J. Polchinski, "Supermembranes," Physics Letters B, Vol. 180, No. 4, 1986, pp. 370-374. http://dx.doi.org/10.1016/0370-2693(86)91204-9

[23] S. Bellucci, E. Ivanov and S. Krivonos, "Superbranes and Super Born-Infeld Theories from Nonlinear Realizations," Nuclear Physics B-Proceedings Supplements, Vol. 102-103, 2001, pp. 26-41. http://dx.doi.org/10.1016/S0920-5632(01)01533-X

[24] J. Hughes and J. Polchinski, "Partially Broken Global Supersymmetry And The Superstring," Nuclear Physics B, Vol. 278, No. 1, 1986, pp. 147-169. http://dx.doi.org/10.1016/0550-3213(86)90111-2

[25] E. Ivanov and S. Krivonos, " $N=1, D=2$ SupermemBrane In The Coset Approach," Physics Letters B, Vol. 453, No. 3-4, 1999, pp. 237-244. http://dx.doi.org/10.1016/S0370-2693(99)00314-7

[26] S. Bellucci, E. Ivanov and S. Krivonos, "Superworldvolume Dynamics of Superbranes From Nonlinear Realizations," Physics Letters B, Vol. 482, No. 1-3, 2000, pp. 233240. http://dx.doi.org/10.1016/S0370-2693(00)00529-3

[27] S. Coleman, J. Wess and B. Zumino, "Structure of Phenomenological Lagrangians I,” Physical Review, Vol. 177, No. 5, 1969, pp. 2239-2247. http://dx.doi.org/10.1103/PhysRev.177.2239

[28] C. Callan, S. Coleman, J. Wess and B. Zumino, "Structure of Phenomenological Lagrangians II,” Physical Review, Vol. 177, No. 5, 1969, pp. 2247-2250. http://dx.doi.org/10.1103/PhysRev.177.2247

[29] D. V. Volkov and J. Sov, "Phenomenological Lagrangians," Soviet Journal of Particles \& Nuclei, Vol. 4, 1973, p. 3.

[30] G. Papadopoulos and P. K. Townsend, "Intersecting MBranes,” Physics Letters B, Vol. 380, No. 3-4, 1996, pp. 273-279. http://dx.doi.org/10.1016/0370-2693(96)00506-0
[31] M. Berkooz, M. Douglas and R. Leigh, "Branes InterSecting At Angles,” Nuclear Physics B, Vol. 480, No. 1-2, 1996, pp. 265-278. http://dx.doi.org/10.1016/S0550-3213(96)00452-X

[32] N. Ohta and P. K. Townsend, "Supersymmetry of MBranes at Angles,” Physics Letters B, Vol. 418, No. 1-2, 1998, pp. 77-84. http://dx.doi.org/10.1016/S0370-2693(97)01396-8

[33] J. P. Gauntlett and C. M. Hull, "BPS States with Extra Supersymmetry,” Journal of High Energy Physics, Vol. 2000, 2000. http://dx.doi.org/10.1088/1126-6708/2000/01/004

[34] J. P. Gauntlett, G. W. Gibbons, C. M. Hull and P. K. Townsend, "BPS States of D=4, N=1 Supersymmetry," Communications in Mathematical Physics, Vol. 216, No. 2, 2001, pp. 431-459. http://dx.doi.org/10.1007/s002200000341

[35] I. Bandos and J. Lukierski, "Tensorial Central Charges and New Superparticle Models with Fundamental Spinor Coordinates,” Modern Physics Letters A, Vol. 14, No. 19, 1999, p. 1257. http://dx.doi.org/10.1142/S0217732399001358

[36] I. Bandos, J. Lukierski and D. Sorokin, "Superparticle Models with Tensorial Central Charges,” Physical Review D, Vol. 61, No. 4, 2000, Article ID: 045002. http://dx.doi.org/10.1103/PhysRevD.61.045002

[37] F. Delduc, E. Ivanov and S. Krivonos, “1/4 Partial BreakIng Of Global Supersymmetry And New Superparticle Actions,” Nuclear Physics B, Vol. 576, No. 1-3, 2000, pp. 196-218. http://dx.doi.org/10.1016/S0550-3213(00)00106-1

[38] S. Fedoruk and V. Zima, "Massive Superparticle with Tensorial Central Charges,” Modern Physics Letters A, Vol. 15, No. 37, 2000, p. 2281. http://dx.doi.org/10.1142/S0217732300002875

[39] S. Bellucci, A. Galajinsky, E. Ivanov and S. Krivonos, "Quantum Mechanics of a Superparticle with 1/4 Supersymmetry Breaking,” Physical Review D, Vol. 65, No. 10, 2002, Article ID: 104023. http://dx.doi.org/10.1103/PhysRevD.65.104023 